\title{
Delincuencia violenta, abuso y no consumo de drogas en adolescentes con riesgo de reincidencia
}

Violent crime, abuse and non-use of drugs in adolescents at recidivism risk

\author{
Joana Calero Plaza (D) http://orcid.org/0000-0002-0999-4123 \\ Universidad Católica de Valencia, España, joana.calero@ucv.es \\ José Manuel Tomás (D http://orcid.org/0000-0002-3424-1668 \\ Universidad de Valencia, España, jose.m.tomas@uv.es
}

José-Javier Navarro-Pérez* (D http://orcid.org/0000-0001-6363-7154

Universidad deValencia, España,J.Javier.Navarro@uv.es

Marcelo Viera (D) http://orcid.org/0000-0003-1826-6440

Universidad Católica de Valencia, España,am.viera@ucv.es

*Autor para

correspondencia :

José-Javier Navarro-Pérez

J.Javier.Navarro@uv.es

Recepción: 27/02/2020

Aprobación: 26/05/2020

Publicación: 22/10/2020

\begin{abstract}
We present an original study on drug use/withdrawal in adolescents, and its association with the risk of violent recidivism. A sample of 192 participants answered the SAVRY questionnaire Structured Assessment of Violence Risk in Youth-, with the aim of analyzing the association between the risk of recidivism in violent crimes involving drug use and withdrawal. The results showed that non-consumption is associated to risk of recidivism in violent intrafamily crimes; the consumption of cocaine was associated to theft with violence and intimidation, while consumption of various drugs to recidivism risk in several crimes, being battery quite common. We conclude that family communication and community prevention programs are extremely important to strengthen positive socialization and develop protective factors to avoid misfits and violent crimes.
\end{abstract}

Key words: juvenile delinquency, types of crime, drug use/nonconsumption, risk assessment, recidivism.

Resumen: Presentamos un estudio original sobre consumo/abstinencia de drogas en adolescentes y su asociación con el riesgo de reincidencia violenta. A una muestra de 192 sujetos se le aplicó el cuestionario SAVRY -Structured Assessment of Violence Risk in Youth-, con el objetivo de analizar la asociación entre el riesgo de reincidencia en delitos violentos y el consumo y abstinencia de drogas. Los resultados mostraron que: el no consumo se asocia al riesgo de reincidencia en delitos violentos intrafamiliares, el consumo de cocaína a robo con violencia e intimidación, y el policonsumo de drogas al riesgo de reincidencia en diferentes delitos, siendo común el ataque contra las personas. Las conclusiones exponen la importancia de la comunicación intrafamiliar y los programas comunitarios de prevención para fortalecer la socialización positiva y desarrollar factores de protección que eviten conductas inadaptadas y delitos violentos.

Palabras clave: delincuencia juvenil, tipos de delito, consumo/no consumo de drogas, evaluación del riesgo, reincidencia. 


\section{Introducción $^{1}$}

Los factores que influyen en la comisión de delitos juveniles han sido ampliamente estudiados; resulta relevante conocer las asociaciones que inciden en los comportamientos delictivos del adolescente, para evaluar el riesgo de reincidencia con mayor precisión y establecer intervenciones que mitiguen los procesos disruptivos y asegurar el bienestar social. El presente estudio muestra la analogía del consumo o abstinencia con la estimación del riesgo de reincidencia delictiva con uso de violencia.

Encontramos investigaciones que han estudiado la relación entre consumo de drogas y conductas delictivas (Miller et al., 2015; Nordstrom y Dackis, 2011) aunque estas no establecen una vinculación clara entre este consumo y la comisión de delitos, de manera que no confirman si dicho consumo puede ser un predictor o activador del comportamiento delictivo o consecuencia de la propia actividad criminal (Menard et al., 2006). La literatura científica no se pone de acuerdo en esta cuestión; sin embargo, hallamos trabajos anteriores que concluyen que el consumo de drogas es el factor que desencadena la conducta delictiva o criminal (Brook et al., 1996; McLaughlin et al., 2000). Otras investigaciones señalan lo contrario: la inexistencia de una relación decisiva entre comisión del delito y uso o abuso de drogas (RecheaAlberola y Fernández-Molina, 2000; Rodríguez-Costas, 1999).

Según diversos estudios, el comportamiento violento se relaciona con diferentes factores de riesgo: los análisis de Millán-Valenzuela y PérezAchundia (2019) encuentran conexión entre pobreza, educación y delincuencia. Algunos estudios planteados desde el modelo explicativo de la teoría intergeneracional de la violencia consideran que haber presenciado conductas agresivas en edades tempranas dentro del entorno de socialización (familia, escuela, amigos...) puede promover un aprendizaje de la violencia (Buehler et al., 2006; Calvete et al., 2015). Sabemos que la custodia compartida en casos de divorcio afecta la comunicación intrafamiliar (Ruiz-Callado y Alcázar, 2019) o que modelos de apego inseguro entre padres e hijos (Arias, 2013; Pons-Salvador, 2007), familias monoparentales (Ibabe et al., 2007) o estilos parentales negligentes (Underwood et al., 2011) también afectan

1 El presente artículo parte de una línea de análisis de la investigación realizada por Marcelo Viera Abelleira presentada en la tesis doctoral titulada "Evaluación del riesgo de reincidencia en adolescentes en conflicto con la ley sometidos a medidas de internamiento judicial por la comisión de delitos violentos", dirigida por la doctora Joana Calero Plaza y el doctor JoséJavier Navarro-Pérez, y leída por su autor el 15 de julio de 2017 en la Universidad Católica de Valencia, ante tribunal público, y cuya calificación fue de Excelente *Cum Laude*. 
a estos procesos. Incluso las fracturas con la escuela y las experiencias de bullying no permiten una socialización completa (Merrell et al., 2006; Raitanen et al., 2017).

En esta línea, hay investigaciones que demuestran una relación entre haber sido víctima de violencia activa y pasiva, y posteriormente haber sido responsable de agresiones violentas tanto de género (Martínez et al., 2016) como de violencia filioparental (Acuña y Fernández-Monroy, 2009; Contreras y Cano, 2016; Sánchez-Heras et al., 2010), entre otros tipos penales.

$\mathrm{Al}$ abordar el uso que los adolescentes hacen de las sustancias adictivas y su posible vinculación con comportamientos delictivos, encontramos estudios que explican cómo se produce el paso del uso hacia la dependencia, y cómo va incrementándose su tolerancia, naturalizándola en sus contextos de relación y socialización -escuela, trabajo, redes de relación, etcétera(Skara et al., 2008). El aumento de comportamientos violentos se vincula con la extensión de los modelos de ocio relacionados con el consumo de alcohol y drogas (Anderson et al., 2007). Los entornos poco normativos, con escasa supervisión adulta y ajenos a las normas de control social, posibilitan la aparición descontrolada de drogas en la esfera cotidiana del adolescente (Doran et al., 2012).

Investigaciones originales indican que la relación entre consumo de drogas y delito depende del ajuste social del adolescente consumidor con su entorno; es decir, a mayor ajuste menor consumo y, por tanto, patrones de comportamiento más estables; y a menor ajuste social, más consumo y patrones de conducta más violentos e inadaptados (Uceda et al., 2016). En este sentido, la relación entre droga y delito se asocia con: comportamientos antisociales, grupos étnicos desfavorecidos, bajo ajuste familiar y antecedentes penales en el núcleo familiar próximo (Sexton y Turner, 2010), vinculación con entornos marginales y la exclusión social (Pierce et al., 2017).

Las relaciones interpersonales que se producen entre adolescentes que consumen drogas son un factor que se ha asociado con la delincuencia juvenil (Melotti y Passini, 2018). Los grupos de adolescentes que residen en entornos de exclusión, con bajo control parental, sin redes de apoyo ni vínculos positivos y con escasa capacidad para resolver conflictos, afianzan prácticas que combinan violencia y consumo de drogas (Cueva, 2012). Valenzuela y Larroulet (2010) identifican la probabilidad de reincidencia, aumentada entre una y seis veces, de aquellos delincuentes juveniles consumidores de drogas.

La sustancia que más se relaciona con la comisión delictiva violenta es la cocaína; las investigaciones de Bennett et al. (2008) establecen que tiene 
una vinculación directa con los patrones violentos de determinados delitos; mientras que el cannabis, por ser la sustancia ilegal más consumida entre adolescentes del sistema de justicia juvenil, presenta mayor probabilidad de asociación con el delito (Thoumi, 2015). Los datos del último informe del Observatorio Español de Drogas y Adicciones (OEDA, 2018) nos indican que la prevalencia en España es la siguiente: consumieron cannabis 26,3\% de los adolescentes y jóvenes de entre 15 y 24 años, cifra ligeramente superior que la media europea, que se sitúa, según el OEDT (2017), en 21,7\%.

Respecto al alcohol, el OEDT (2017) señala que es la sustancia legal más consumida por adolescentes: 76,9\%; cuestión que constituye un factor de riesgo esencial en su asociación con delitos violentos, pues a su vez es la cuarta en la escala de sumisión (Nutt et al., 2007) y aumenta hasta cinco veces la probabilidad de riesgo de reincidencia en la comisión de delitos con violencia cuando ha sido consumida (Livingston, 2014). Un estudio de Vilalta (2010) en la Ciudad de México coincidía con esta misma cuestión.

La investigación científica de Nutt et al. (2007) informó que las sustancias que generan mayor dependencia, en este orden, son: heroína, cocaína, barbitúricos y alcohol. Por último, el policonsumo (tres o más sustancias) es un factor de riesgo en la reincidencia delictiva adolescente (Scheidell et al., 2018), teniendo presente que el consumo permite asociar con qué tipo de drogas se incurre en mayor o menor correlación con los delitos.

La evaluación de la reincidencia delictiva en adolescentes es un tema de amplio debate y réplica. Son muchas las investigaciones que inciden en conocer los elementos que interaccionan en la asunción de comportamientos delictivos con uso de violencia por parte de adolescentes (Pierce et al., 2017; Rechea y Fernandez-Molina, 2000; Skara et al., 2008) En este sentido, es importante señalar que en la valoración de riesgo intervienen variables del tipo: territorio de socialización, grupo de iguales, exposición a violencia previa, recursos y redes, y el consumo de drogas.

Los sistemas penales juveniles establecen grandes diferencias entre los delitos perpetrados mediante el uso de violencia (Lindquist, 2016). Es cierto que existen conductas antisociales que no necesariamente exigen un comportamiento violento: delitos de falsedad documental, contra la salud pública, estafa, hurto, etcétera. Pero también los hay que sí. Estos podrían dividirse entre los que se cometen contra las cosas (robo con fuerza o delitos contra el patrimonio y orden socioeconómico, incendio intencional...) y los que se cometen contra el bien jurídico protegido que goza de mayores garantías: la vida o intimidad de las personas (robo con violencia, amenazas, agresión sexual, lesiones o violencia filioparental, entre otros). La Ley Orgánica de Responsabilidad Penal de los Menores 5/2000 (Ministerio de 
Justicia, 2000) en España es más sensible con los delitos contra las personas, porque según De Vicente (2018) implican comportamientos que, por la naturaleza del bien jurídico vulnerado, son considerados de mayor gravedad. Por tanto, los delitos con uso de violencia presentan mayor preocupación jurídica, pues además afectan a la vida y el control social.

Actualmente existen distintos instrumentos que son capaces de evaluar el riesgo y predecir la actividad criminal. Los más utilizados son el Inventario de Gestión e Intervención para Jóvenes (Youth Level Service/ Case Management Inventory) (Hoge y Andrews, 2006) y la Evaluación estructurada del riesgo de violencia en la juventud (Structured Assesment of Violence Risk in Youth [SAVRY]) (Borum et al., 2003). Los investigadores que estudian la delincuencia violenta consideran al SAVRY como herramienta referencial (Lodewijks et al., 2010; Navarro-Pérez et al., 2020; Schmidt et al., 2011). El SAVRY es un cuestionario que incluye factores de protección que interaccionan con variables de riesgo; además, permite identificar diferentes niveles de riesgo y colabora en la creación de programas dependiendo del nivel de vulnerabilidad del sujeto.

La presente investigación intenta identificar la posible correlación entre no consumo/consumo-policonsumo con la estimación del riesgo de cometer prácticas delictivas violentas. Para ello, se plantean tres hipótesis:

H1 La ausencia de consumo de drogas por parte de los adolescentes se asocia al riesgo de reincidencia en delitos de violencia filioparental.

H2 El consumo de cocaína se asociaría con mayor probabilidad de cometer el delito de robo con violencia e intimidación.

H3 El policonsumo de drogas, como alcohol, cannabis o cocaína, se relacionaría con diferentes delitos caracterizados comúnmente por el uso de la violencia

\section{Método}

\section{Participantes y procedimiento}

La muestra se compone de 192 adolescentes ingresados por resolución judicial en Centros de Internamiento de Medidas Judiciales en la Comunidad Valenciana (España); $77 \%$ son varones y 23\%, mujeres.

Los criterios de inclusión de los sujetos participantes de la muestra son:

- Edad: desde los 14 hasta los 21 años.

- Adolescentes sometidos a medidas de internamiento judicial con sentencia firme.

- Comisión de delitos con uso de violencia. 
El desarrollo de esta investigación se llevó a cabo de acuerdo con las recomendaciones de los comités de ética de las instituciones participantes y cumplió con los estándares nacionales -Ley 14/2007, de 3 de julio, de Investigación biomédica- (Jefatura del Estado, 2007), e internacionales de ética en investigación. Los resultados expuestos son extraídos de una investigación más amplia. El presente estudio fue aprobado sin objeciones por la Comisión de Ética e Investigaciones Experimentales; y las personas que cumplieron con los criterios de inclusión recibieron previamente la información necesaria, dieron su consentimiento de participación voluntaria por escrito y consentimiento informado a los tutores legales, conforme a la Declaración de Fortaleza (2013). Asimismo, se explicó a los y las participantes la posibilidad de interrumpir o rechazar su participación en cualquier momento.

\section{Instrumentos}

El principal instrumento utilizado en esta investigación fue la Evaluación estructurada del riesgo de violencia en la juventud (SAVRY) (Borum et al., 2003) con la adaptación española de Vallés y Hilterman (2006). Esta es una herramienta que predice la reincidencia delictiva usando 30 variables, de las que 24 son de riesgo y seis de protección; además, presenta amplia réplica por la comunidad científica (Lodewijks et al., 2008; Navarro-Pérez y Pastor, 2017; Shepherd et al., 2014; Ortega-Campos et al., 2017), en especial cuando se trata de adolescentes privados de la libertad, como en este estudio (Gammelgard et al., 2008; Klein et al. 2012).

De los factores de riesgo, diez se refieren al pasado del adolescente, seis son contextuales y ocho, individuales. Los riesgos se califican desde bajo (0) hasta alto riesgo (2). Los factores de protección se califican como presente (1) o ausente (0). Para este trabajo se ocupó el indicador de factor de riesgo SAVRY sobre consumo de drogas, y adicionalmente se aplicaron factores demográficos como consumo/ abstinencia, tipo de droga consumida y tipo de delito perpetrado, para poder establecer vínculos entre delitos cometidos con violencia y consumo/abstinencia y tipo de droga. Asimismo, para el presente estudio, SAVRY permite predecir el riesgo de reincidencia delictiva, para después conectarlo tanto con el consumo /abstinencia de tipos de drogas como con la inmersión en conductas delictivas con uso de violencia. 


\section{Análisis estadistico}

Se calcularon las frecuencias para todas las variables en estudio. Las relaciones entre los indicadores de consumo de drogas y el tipo de delito se analizaron con pruebas de independencia de chi-cuadrado, aplicadas a las tablas de contingencia. La prueba de chi-cuadrado de Pearson usualmente utiliza la aproximación de chi-cuadrado, pero en este caso se calculó un valor $p$ exacto usando la distribución de probabilidad verdadera. Las relaciones se declararon estadísticamente significativas cuando $\mathrm{p}<.05$. Adicional a la prueba de chi-cuadrado, se estimó una medida del tamaño del efecto para cada prueba, en este caso la V (V) de Cramer. Los valores de corte habituales para declarar los efectos como medianos o grandes cuando los grados de libertad son 5 o mayores son $.04, .13$ y .22 (Cohen, 1988).

Para facilitar la interpretación de las tablas de contingencia, se calcularon los porcentajes por fila y los residuos estandarizados. El residual estandarizado es una medida de la fuerza de la diferencia entre los valores observados y los valores esperados, y miden la significación de las celdillas para el valor de chi-cuadrado. El residuo estandarizado permite identificar qué celdillas son precisamente las que más contribuyen a la asociación encontrada y las que menos. La regla general para determinar qué significa el residuo estandarizado es: residuos menores que -2 , la frecuencia observada de la celdilla es menor que la frecuencia esperada (bajo la hipótesis nula) y residuos mayores que 2, la frecuencia observada es mayor que la frecuencia esperada. Cuanto mayor sean los residuos en valor absoluto, mayor será la diferencia entre las frecuencias observadas y las esperadas. Todos los análisis estadísticos se realizaron en SPSS 24.

\section{Resultados}

En esta investigación, las principales variables de interés fueron el tipo de delito violento y el historial de consumo (policonsumo) y no consumo de drogas. La Figura $1^{2}$ muestra los porcentajes para cada tipo de delito cometido por los adolescentes. La mayoría $(57,3 \%)$ cometió delitos de violencia filioparental sobre sus cuidadores familiares, seguidos de robo con fuerza, violencia e intimidación. Otros delitos fueron menos representativos.

2 Todas las figuras y las tablas se encuentran en el Anexo, al final del presente artículo (Nota del editor). 
Respecto al consumo de drogas, la Figura 2 expone, de manera compacta, los porcentajes de los sujetos de la muestra que consumieron varios tipos de sustancias antes de ser detenidos, juzgados y de ingresar en un centro de internamiento. La mayoría de ellos declaró que consumía alcohol con asiduidad. Los análisis realizados informaron del consumo de cannabis con menor asiduidad, pero aun así 69.3\% de ellos declaró que lo hacía usualmente. Mucho menos frecuente fue el consumo de cocaína: 15.7\% ocasional y $13.1 \%$ habitual.

Además, en función de estas frecuencias, se codificó una nueva variable para separar a los adolescentes de la muestra que declararon que nunca consumieron ningún tipo de las sustancias mencionadas, de quienes declararon algún tipo de consumo: $6.8 \%$ nunca consumió sustancias legales o ilegales, mientras que 93,2\% consumió, al menos de forma ocasional, algún tipo de sustancia.

Cuando se comparó a los adolescentes que nunca tomaron drogas con los que declararon que sí las habían consumido respecto a sus tipos de delitos, el chi-cuadrado fue estadísticamente significativo: $\chi^{2}(7)=13.946$, $\mathrm{p}=.03, \mathrm{~V}=.256$. Hubo diferencias entre ambos grupos en la probabilidad de los tipos de delito que cometieron. La Tabla 1 ofrece la clasificación cruzada de las frecuencias de ambos grupos por tipo de delito. Al observar los porcentajes y los residuos estandarizados, los adolescentes que nunca consumieron drogas tuvieron mayor probabilidad de riesgo de reincidencia para atentar contra la autoridad de los padres o cuidadores principales. Los que no consumieron mostraron menor probabilidad de robar con violencia e intimidación que quienes consumieron algún tipo de droga.

El consumo de drogas entre los adolescentes y su relación con el tipo de delito se analizó con más detalle sustancia a sustancia. En primer lugar, se utilizó una prueba de chi-cuadrado de independencia entre el consumo de alcohol y el tipo de delito, y los resultados no fueron significativos: $\chi^{2}(7)=5.077, \mathrm{p}=.656, \mathrm{~V}=.163$. La relación entre el consumo de cannabis y el tipo de delito tampoco fue estadísticamente significativa: $\chi^{2}(7)=5.049$, $\mathrm{p}=.679, \mathrm{~V}=.162$. Sin embargo, cuando se estudió el consumo de cocaína en relación con el tipo de delito, la relación fue estadísticamente significativa y grande: $\chi^{2}(7)=21.379, \mathrm{p}=.009, \mathrm{~V}=.335$. La Tabla 2 ofrece frecuencias, porcentajes y residuos estandarizados. Los grandes residuos mostraron con claridad que quienes consumieron cocaína tuvieron mayores probabilidades (un residuo de 4 ) de cometer un robo con intimidación que aquellos que no la consumieron (un residuo de -4 ). 
Por otro lado, los residuos también mostraron que quienes no consumieron cocaína tuvieron mayor riesgo de reincidencia (residual de 3.1) para cometer violencia filioparental que quienes consumían cocaína (residual de -3.1). Los porcentajes exhibieron el mismo patrón. El 61.4\% de los que no consumieron cocaína cometieron delitos de violencia filioparental, que representa $28 \%$ entre los consumidores de cocaína. En tanto, $48 \%$ de los consumidores de cocaína y sólo $14.5 \%$ de los no consumidores de esta sustancia cometieron robos con violencia e intimidación.

En cuanto al policonsumo de drogas y el tipo de delito cometido, la relación también fue estadísticamente significativa y grande: $\chi^{2}(14)=24.825$, $\mathrm{p}=.036, \mathrm{~V}=.254$. La Tabla 3 muestra los resultados; entre ellos destacan los asociados a altos residuos. Los adolescentes que consumieron alcohol, cannabis y cocaína tuvieron más riesgo de reincidir en carreras delictivas versátiles: violencia de género (residual de 2.4) e intentos de atentado contra la autoridad (residual de 2.6), mientras que fueron menos propensos a cometer un delito intrafamiliar de violencia filioparental (residual de -3.1).

\section{Discusión}

A diferencia de los estudios sobre consumo de drogas y participación delictiva de adolescentes en conflicto con la ley de los años noventa, en los que se encontraba una clara relación entre abuso de drogas y actividad criminal (Brook et al., 1996; Chaiken y Chaiken, 1990), las investigaciones recientes refieren que dicho consumo no puede considerarse como activador aislado del comportamiento antisocial (Menard et al., 2006), ni tampoco de prácticas delictivas caracterizadas por el uso de violencia (Hedström e Ylikoski, 2010).

Estos estudios inciden en desconectar la asociación en espiral droga-delito (Zawadzki, 2007) porque, según describen, la actividad criminal es previa al consumo de drogas y, en segundo término, a medida que se incrementa la asunción de comportamientos criminales, también crece el consumo de drogas; por tanto, existe una retroalimentación y no una causa efecto de uno sobre otro (Walters, 2018). De manera más explícita, Drazowski et al. (2015) informan que "la edad, el sexo, la exposición a la violencia, el nivel socioeconómico más bajo, el mayor consumo de alcohol y los antecedentes de delincuencia son más importantes que el consumo de drogas a efectos de cometer un delito". En esta línea de nuestro trabajo, Yoder y Caserta (2018) consideran de gran interés poder establecer analogías entre la responsabilidad y tipo de infracción asumida por los adolescentes con el abuso de sustancias, y si éstas se relacionan en mayor medida a unos delitos que a otros. 
Respecto a la primera hipótesis, se confirma que los adolescentes no consumidores de droga asumirían un tipo penal de violencia filioparental. Los resultados de la investigación indican que los adolescentes que no consumieron ningún tipo de sustancia adictiva cometieron mayoritariamente delitos asociados a la violencia filioparental. Esta aportación viene a confirmar la definición que, de violencia filioparental, establece Pereira (2006) al apuntar que excluye los casos relacionados con el consumo de tóxicos por resultar aislados. En esta línea, coincidimos con Walsh y Krienert (2007), quienes -con base en una muestra de 17.957 adolescentes infractores de CPV (Child Parent Violence)- concluyeron que un bajísimo porcentaje de estos se encontraba bajo los efectos del alcohol o las drogas en los episodios de responsabilidad penal, siendo además asaltos altamente violentos incluso usando enseres de cocina, pero con lesiones leves. Por tanto, en concordancia con el estudio de Obeida (2012) en Suiza con 829 sujetos, podemos conceptualizar esta delincuencia violenta como de baja intensidad.

Respecto a la segunda hipótesis, planteábamos que el consumo de cocaína se asociaría al tipo penal de robo con violencia, y los resultados mostraron que $48 \%$ de los adolescentes consumidores de cocaína cometieron robos con violencia e intimidación. Los resultados nos permiten confirmar la hipótesis; además, en esta dirección, las investigaciones recientes coinciden con Kuhns y Clodfelter (2009) en que la cocaína es una sustancia aceleradora de la tensión -enlazándola con experiencias de comportamiento violento-, en particular vía intravenosa o aspirada. Un reciente estudio longitudinal de Beharie et al. (2019) señaló que los jóvenes que sufrieron vejaciones en la infancia y presentaron consumo prematuro de cocaína en tiempo prolongado, cometieron durante la adolescencia y juventud delitos violentos contra las personas y las cosas; además, en la edad adulta continuaron con altas tasas de riesgo de reincidencia delictiva. En esta línea, los resultados de Skara et al. (2008) indicaron que los sujetos que mantienen una fuerte dependencia a la cocaína muestran más problemas relacionados con la violencia interpersonal.

$\mathrm{Al}$ atender a la última de las hipótesis de investigación, relativa a validar si el policonsumo de drogas como alcohol, cannabis y cocaína se relacionaría con una mayor asociación a dinámicas delictivas heterogéneas, caracterizadas por el uso de la violencia, se encontró que los adolescentes que las policonsumieron tuvieron más probabilidades de participar en robos con violencia e intimidación, violencia de género y atentados contra la autoridad pública, mientras que fueron menos propensos a cometer un delito de violencia filioparental. 
Así, la intoxicación por alcohol se asocia frecuentemente al comportamiento agresivo y violento (Parrott y Eckhardt, 2018). En un estudio con más de 1420 adolescentes, Melotti y Passini (2018) evidenciaron que las variables sociopsicológicas (es decir, la cultura y actitudes violentas) tienen un importante impacto en la coexistencia entre policonsumo de sustancias y comportamiento violento.

Otro estudio longitudinal con casi 15000 sujetos en Estados Unidos señaló que los niños que sufrieron una infancia traumática tuvieron problemas prácticamente a lo largo de toda su vida, con dependencia a la marihuana y otras drogas, y con la comisión de distintos delitos violentos (Scheidell et al., 2018). Pierce et al. (2017) concluyen que las iniciativas de prevención de policonsumo de alcohol y opiáceos podrían ser efectivas para reducir las tasas de delincuencia violenta. Así, el policonsumo de sustancias como cannabis, alcohol y cocaína incidiría análogamente según nuestros resultados en la asociación a delitos violentos contra las personas. En este sentido, no se puede establecer una relación entre consumo y conducta delictiva, pero sí conectar el riesgo de reincidencia en el tipo penal que el adolescente comete y su nivel de asociación a las drogas - desde el no consumo hasta el policonsumo-.

El aumento en el consumo de sustancias de distinta índole genera graves problemas de control vital; en mayor medida en la vida de adolescentes y jóvenes, afectando su desarrollo cognitivo y enlazándose con carreras delictivas versátiles. Esta representación se produce en procesos delictivos que han superado la fase inicial: es decir, el adolescente que comete delitos y ya ha conectado con la delincuencia prolongada. Tarin y Navarro (2006: 239) definen estos procesos como "fase oculta y cresta de la ola", promoviendo conexiones con otras actividades de alto riesgo como el mismo policonsumo, el contacto con otros delincuentes (Braga, 2016), la delincuencia grupal o lazos con la violencia para salvaguardar el estatus criminal (Lynch et al., 2003). Hay suficiente literatura científica que lo corrobora; incluso algunos estudios han demostrado que en los centros privativos de la libertad, los politoxicómanos que reciben tratamiento mediante fármacos sustitutivos (metadona) siguen abusando sistemáticamente de distintas drogas (Moreno et al., 2016) y extorsionando y violentando a otros jóvenes privados de la libertad (Martínez-Reyes et al., 2019).

En los últimos años, las investigaciones han confirmado que el consumo de diferentes sustancias representa un riesgo tanto para cometer delitos como para aumentar la severidad de los mismos (Pérez y Ruiz, 2017; Viera, 2017); sin embargo, no hay evidencias científicas que demuestren que las drogas sean claramente causa o consecuencia de los hechos delictivos. Ambas temporalidades aparecen conectadas al delito sin una clara inclinación. 
Respecto a los riesgos asociados a la reincidencia, los estudios consultados indican que el consumo de drogas y la violencia son factores de alto impacto en la reproducción de conductas antisociales (Justicia et al., 2006; Menard et al., 2006; Nordstrom y Dackis, 2011). La investigación de Navarro-Pérez et al. (2020) apuntan a los factores de protección como indicadores para predecir el riesgo de reincidencia delictiva. Otro estudio reciente de Ortega-Campos et al. (2020) pone el foco de atención en la importancia de los factores de protección para atajar la desviación social, ya que la adolescencia permite reorientar las circunstancias cotidianas y con ello reducir la delincuencia a un corto periodo vital; y Carbonell y Mestre (2019) inciden en las distorsiones cognitivas.

En esta línea cabe destacar cómo los grupos de investigación internacional subrayan las potencialidades que tienen los adolescentes violentos, dejando en un segundo término sus factores de riesgo. El éxito de los programas de ajuste social viene determinado por estímulos apuntalados en el apoyo social, la implicación escolar, las habilidades para resistir y hacer frente a los grupos de riesgo. Navarro-Pérez y Pastor (2017) confirman que la formación y la motivación hacia competencias laborales disminuye los riesgos de reincidencia delictiva, porque capacitan al adolescente para enfrentarse a las dificultades. Por tanto, los factores de protección se presentan como estandarte para reducir las tasas de delito y con ello abordar la delincuencia violenta adolescente con mayores estrategias para su seguimiento y control.

Los resultados de la investigación arrojan luz en el ámbito profesional y orientan hacia el diseño de programas de prevención, consumo responsable o deshabituación, evaluando la asociación entre violencia, responsabilidad penal y consumo/abstinencia de drogas como un cóctel necesariamente abordable para favorecer el bienestar y la seguridad colectiva (De Souza et al., 2018). Además, estos resultados ponen el acento en el no consumo como factor que contribuye a reducir la delincuencia violenta, entendiendo la violencia filioparental como tipo penal de baja intensidad (Walsh y Krienert, 2007). Por tanto, se debe orientar en programas donde se refuerce o fortalezca el no consumo como actividad preventiva frente al delito y al uso de la violencia en general. Los estudios de Beharie et al. (2019) destacan la importancia de trabajar la abstinencia, con el fin de disminuir los niveles de delincuencia violenta, que incluso pueden prolongarse hasta la edad adulta. Se establece la necesidad de colaborar con las cortes penales juveniles en la predicción del riesgo de reincidencia con uso de violencia. Los programas de tratamiento penal podrían incidir en estas cuestiones; en primer lugar y referido a los 
delitos contra las personas, como mecanismo de protección hacia las víctimas y al mismo tiempo como elemento disuasorio para los delincuentes juveniles y optar por decisiones correctas (Bavolar y Bacikova-Sleskova, 2020).

En segundo lugar, en cuanto a las actuaciones de versatilidad en la comisión delictiva, los resultados sugieren que tal vez pueda considerarse esta dimensión en la asociación entre policonsumo y actividad criminal diversa, a efectos de potenciar la conciencia del delincuente sobre el análisis funcional que conecta con el delito. En esta línea, los programas de entrenamiento cognitivo permiten anticipar respuestas. Los trabajos de Raitanen et al. (2017), mediante propuestas narrativas, posibilitan adelantar las consecuencias de determinadas conductas desviadas en la estructura de pensamiento de adolescentes violentos, procedentes de entornos de exclusión. Estas reflexiones cognitivas permiten desarrollar la empatía de los adolescentes a partir de sus propias experiencias violentas, anticipándose desde una perspectiva crítica (Barry, 2016) a las consecuencias derivadas de un uso irresponsable de su libertad. Mediante estas actuaciones, los adolescentes son capaces de identificar que el consumo de drogas tiene una consecuencia directa con actividades ilícitas y violentas; por tanto, conectar reflexivamente con las previsibles consecuencias, les permite ser más conscientes de su realidad y de los riesgos asociados a ella.

En sintonía con diferentes estudios nacionales e internacionales (Arias, 2013; Del Moral et al., 2015; Justicia et al., 2006; Justicia y Cantón, 2011; Montero et al., 2020), los resultados obtenidos en esta investigación nos impelan al diseño de programas de prevención orientados a la familia y la escuela, estableciendo como objetivos prioritarios aquellos que posibiliten impactar en el pensamiento y fomentar las habilidades de comunicación interpersonal entre padres e hijos, además de otras herramientas que permitan empoderar a los adolescentes hacia la responsabilidad y la socialización positiva.

\section{Conclusiones}

La presente investigación conecta los tres conceptos (abuso/no consumo de droga, riesgo de reincidencia y violencia como connotación reprobable). Profundiza tanto en factores protectores, variables cognitivo-reflexivas del pensamiento crítico como en herramientas para identificar y establecer programas reeducativos, resocializadores, con el propósito de reconducir las trayectorias vitales delictivas con uso de violencia y abuso/no consumo de drogas. 
En definitiva, entendemos necesaria la incorporación de lineamientos que incidan en el apoyo psicosocial para reducir la vulnerabilidad, de programas de prevención comunitaria en el uso y abuso de drogas, con el fin de robustecer factores de protección e incrementar las fortalezas en los adolescentes.

\section{Referencias}

Acuña, Carmen y Fernández-Monroy, Ana (2009), "Violencia filioparental. Madres Victimas-Hijos Victimarios", en TS Cuadernos de Trabajo Social, núm. 5, Chile: Universidad de San Sebastián.

Anderson, Zara et al. (2007), "Alcohol, party and violence", en Journal of Sociology of Violence, vol. 7, núm. 3, Reino Unido: Dickinson.

Arias, Walter (2013), "Agresión y violencia en la adolescencia: La importancia de la familia", en Avances en Psicología, vol. 21, núm. 1, Perú: Universidad Femenina del Sagrado Corazón.

Barry, Mary (2016), "On the cusp of recognition: Using critical theory to promote desistance among young offenders”, en Theoretical Criminology, vol. 20, núm. 1, Estados Unidos: Sage.

Bavolar, Josef y Bacikova-Sleskova, María (2020), "Psychological protective factors mediate the relationship between decision-making styles and mental health", en Current Psychology, vol. 39, núm. 4, Estados Unidos: Springer.

Beharie, Nisha et al. (2019), "Associations of Adolescent Exposure to Severe Violence with Substance Use from Adolescence into Adulthood: Direct Versus Indirect Exposures”, en Substance Use \& Misuse, vol. 54, núm. 2, Reino Unido: Taylor \& Francis.

Borum, Randy et al. (2003), SAVRY Structured Assessment of Violence Risk in Youth, Estados Unidos: University of South Florida y Florida Mental Health Institute.

Braga, Anthony (2016), Gun Violence Among Serious Young Offenders, Estados Unidos: Office of Community-Oriented Policing Services.

Brook, Judith et al. (1996), Young Adult Drug Use and Delinquency: Childhood Antecedents and Adolescent Mediators", en Journal of the American Academy of Child \& Adolescent Psychiatry, vol. 35, núm. 12, Estados Unidos: Elsevier.

Buehler, Cheryl et al. (2006), "Hostilidad interparental y comportamiento problemático en la adolescencia temprana: el papel mediador de aspectos específicos de la crianza de los hijos", en Revista de Investigación sobre la Adolescencia, vol. 16, núm. 2, España: Universitat Politècnica de València.

Calvete, Esther et al. (2015), "Child-to-parent violence: An exploratory study of the roles of family violence and parental discipline through the stories told by Spanish children and their parents", en Violence and Victims, vol. 30, núm. 6, Estados Unidos: Springer.

Carbonell, Ángela y Mestre, María Vicenta (2019), “Sexismo, amor romántico y desigualdad de género. Un estudio de adolescentes latinoamericanos residentes en España”, en América Latina Hoy, vol. 83, España: Servicio de Publicaciones de la Universidad de Salamanca.

Chaiken, Jhon y Chaiken, Mary (1990), “Drugs and Predatory Crime”, en Crime and Justice, vol. 13, Estados Unidos: The University of Chicago Press. 
Joana Calero Plaza, José Manuel Tomás, José-Javier Navarro-Pérez y Marcelo Viera. Delincuencia violenta, abuso y no consumo de drogas en adolescentes con riesgo de reincidencia

Cohen, John (1988). Statistical power analñysis for de behavioral sciences. New York: LEA.

Contreras, Lourdes y Cano, María Carmen (2016), "Violencia filio-parental: el papel de la exposición a la violencia y su relación con el procesamiento sociocognitivo", en The European Journal of Psychology Applied to Legal Context, vol. 8, núm. 2, España: Colegio Oficial de Psicólogos de Madrid.

Cueva, Gloria (2012), "Violencia y adicciones: problemas de salud pública”, en Revista Peruana de Medicina de Salud Pública, vol. 29, núm. 1, Perú: Instituto Nacional de Salud.

Declaración de Fortaleza (2013), "Principio ético para las investigaciones médicas en seres humanos", ratificado en la 64a Asamblea en Fortaleza (Brasil), Francia: Wold Medical Association.

De Souza, Luis Fernando et al. (2018), "Necropolítica e Pesquisa-Intervenção sobre Homicídios de Adolescentes e Jovens em Fortaleza”, en Psicologia: Ciência e Profissão, vol. 38, núm. 2. DOI: 10.1590/1982-3703000212908 Disponible en: https://www. scielo.br/scielo.php?script=sci_arttext\&pid=S1414-98932018000600192\&tlng=pt [ 31 de enero de 2020].

De Vicente, Rosario (2018), Vademecum del Derecho Penal, España: Tirant lo Blanch.

Del Moral, Gonzalo et al. (2015), "Concepciones sobre la violencia filio-parental en servicios sociales: Un estudio exploratorio”, en Acción Psicológica, vol. 12, núm. 1, España: Universidad Nacional de Educación a Distancia.

Doran, Neal et al. (2012), "Adolescent Substance Use and Aggression: A Review”, en Criminal Justice \& Behaviour, vol. 39, núm. 6, Estados Unidos: Sage.

Drazowski, Tess et al. (2015), "Use of prescription drugs and future delinquency among adolescent offenders", en Journal of substance abuse treatment, vol. 48, Estados Unidos: Elsevier.

Gammelgard, Mónica et al. (2008), "The predictive validity of the Structured Assessment of Violence Risk in Youth (SAVRY) among institutionalized adolescents”, en Journal of Forensic Psychiatry Psychology, vol. 19, Reino Unido: Routledge.

Hedström, Peter y Ylikoski, Petri (2010), "Causal mechanisms in the social sciences”, en Annual Review of Sociology, vol. 36, Estados Unidos: Annual Reviews.

Hoge, Robert y Andrews, Don (2006), Youth Level of Service/ Case Management Inventory (YLS/CMI), Canadá: Multi-Health Systems.

Ibabe, Izaskun et al. (2007), Violencia filio-parental: Conductas violentas de jóvenes hacia sus padres, España: Servicio Central de Publicaciones del Gobierno Vasco.

Jefatura del Estado (2007), "Ley 14/2007 de Investigación biomédica”, en Boletín Oficial del Estado, núm. 159, España: Gobierno de España.

Justicia, Fernando et al. (2006), "Aproximación a un nuevo modelo explicativo del comportamiento antisocial”, en Revista Electrónica de Investigación Psicoeducativa, vol. 4, núm. 2, España: Universidad de Almería.

Justicia, Fernando y Cantón, José (2011), “Conflictos entre padres y conducta agresiva y delictiva en los hijos”, en Psicothema, vol. 23, núm. 1, España: Universidad de Oviedo.

Klein, Verena et al. (2012), "Assessment of accused juvenile sex offenders in Germany: a comparison of five different measures", en Behaviour Sciences Law, vol. 30, Estados Unidos: John Wiley \& Sons.

Kuhns, Joseph y Clodfelter, Tammatha (2009), "Illicit drug-related psychopharmacological violence: The current understanding within a causal context", en Aggression and Violent Behavior, vol. 14, Estados Unidos: Elsevier. 
Lindquist, Ricardo (2016), "El proceso formativo-readaptativo para menores infractores: un acercamiento al panorama mexicano", en Revista de Investigaciones UCM, vol. 16, núm. 27, España: Universidad Complutense de Madrid.

Livingston, Mark (2014), "Trends in non-drinking among Australian adolescents", en Addiction, vol. 109, Reino Unido: John Wiley \& Sons.

Lodewijks, Henny et al. (2008), "Predictive validity of the Structured Assessment of Violence Risk in Youth (SAVRY) during residential treatment", en International Journal of Law and Psychiatry, vol. 31, Canadá: Elsevier.

Lodewijks, Henny et al. (2010), "Reoffending: A study in three samples of adolescent offenders. The impact of protective factors in desistance from violent", en Journal Interpersonal Violence, vol. 25, Estados Unidos: Sage.

Lynch, Mary et al. (2003), "Youth justice: criminal trajectories", en Trends \& Issues in Crime and Criminal Justice, núm. 265, Australia: Australian Institute of Criminology.

Martínez, Jorge et al. (2016), "Relación entre la violencia en el noviazgo y observación de modelos parentales de maltrato", en Psychologia: Avances de la Disciplina, vol. 10, núm. 1, Colombia: Universidad San Buenaventura.

Martínez-Reyes, Alberto et al. (2019), "Adolescentes en las pandillas salvadoreñas: de niños desprotegidos a criminales violentos", en América Latina Hoy, vol. 83, España: Universidad de Salamanca.

McLaughlin, Colleen et al. (2000), "The relationship between substance use, drug selling, and lethal violence in 25 juvenile murderers", en Journal of Forensic Sciences, vol. 45, Estados Unidos: American Academy of Forensic Sciences.

Melotti, Giannino y Passini, Stefano (2018), "Drug Use and Violence Among Adolescents: The Mediation Effect of Attitudes Supporting Violence", en Journal of Child \& Adolescent Substance Abuse, vol. 27, núm. 4, Reino Unido: Taylor \& Francis.

Menard, Scott et al. (2006), "Drugs and crime revisited", en Justice Quarterly, núm. 29, Estados Unidos: Routledge.

Merrell, Kenneth et al. (2006), "Relational aggression in children and adolescents: A review with implications for school settings", en Psychology in the Schools, vol. 43, Reino Unido: Wiley-Blackwell.

Millán-Valenzuela, Henio y Pérez-Achundia Eduardo (2019), "Educación, pobreza y delincuencia: ¿nexos de la violencia en México?”, en Convergencia Revista de Ciencias Sociales, núm. 80, México: Universidad Autónoma del Estado de México.

Miller, Peter et al. (2015), "Illicit drug use and experience of harm in the night-time economy", en Journal of Substance Use, vol. 20, núm. 4, Reino Unido: Taylor \& Francis.

Ministerio de Justicia (2000), "Ley Orgánica de Responsabilidad Penal de los Menores 5/2000", en Boletin Oficial del Estado, núm. 11, España: Gobierno de España.

Montero, Pablo et al. (2020), "Adolescent substance use and its association with risk and protective factors. An exploratory analysis of the large-scale school survey of Comunidades Que se Cuidan, Colombia”, en Adicciones, vol. 32, núm. 2, España: Sociedad Científica Española de Estudios sobre el Alcohol, el Alcoholismo y las Toxicomanías.

Moreno, Luis et al. (2016), "Impulsividad en varones con prescripción de benzodiacepinas y metadona en prisión", en Adicciones, vol. 28, núm. 4, España: Sociedad Científica Española de Estudios sobre el Alcohol, el Alcoholismo y las Toxicomanías. 
Joana Calero Plaza, José Manuel Tomás, José-Javier Navarro-Pérez y Marcelo Viera. Delincuencia violenta, abuso y no consumo de drogas en adolescentes con riesgo de reincidencia

Navarro-Pérez, José Javier y Pastor-Seller, Enrique (2017), "Factores dinámicos en el comportamiento de delincuentes juveniles con perfil de ajuste social. Un estudio de reincidencia”, en Psychosocial Intervention, vol. 26, núm. 1, España: Elsevier.

Navarro-Pérez, J.J. et al. (2020), "Factors in Assessing Recidivism Risk in Young Offenders", en Sustainability, vol. 12, Estados Unidos: Multidisciplinary Digital Publishing Institute.

Nordstrom, Benjamin y Dackis, Charles (2011), "Drugs and Crime", en The Academic Journal of Psychiatry \& Law, vol. 39, núm. 4, Estados Unidos: Academy of Psychiatry and the Law.

Nutt, David et al. (2007), "Development of a rational scale to asses that harm of drugs of potential misuse”, en The Lancet, vol. 369, Reino Unido: Lancet Publishing Group \& Elsevier.

Obeida, Zeinab (2012), "Perfiles de delincuentes especializados y delincuentes versátiles estudio cuantitativo basados en la información del ISRD-21 Suiza”, en Justicia Juris, vol. 8, núm. 2, Colombia: Universidad Autónoma del Caribe.

OEDT (Observatorio Europeo de las Drogas y las Toxicomanías) (2017), "Informe Europeo sobre drogas 2017. Tendencias y novedades”, en Oficina de Publicaciones de la Unión Europea, Luxemburgo: Publicaciones de la Unión Europea.

OEDA (Observatorio Español de las Drogas y las Adicciones) (2018), "Informe 2018. Alcohol, tabaco y drogas ilegales en España”, en Plan Nacional sobre Drogas, España: Ministerio de Sanidad, Consumo y Bienestar Social.

Ortega-Campos, Elena et al. (2017), "The Predictive Validity of the Structured Assessment of Violence Risk in Youth for Young Spanish Offenders", en Frontiers Psychology, vol. 8, Suiza: Frontiers.

Ortega-Campos, Elena et al. (2020), "Assessing the Interactions between Strengths and Risk Factors of Recidivism through the Structured Assessment of Violence Risk in Youth (SAVRY)", en International Journal Environmental Research \& Public Health, vol. 17, Suiza: Multidisciplinary Digital Publishing Institute.

Parrott, Dominic y Eckhardt, Christopher (2018), "Effects of alcohol on human aggression", en Current Opinion in Psychology, vol. 19, Estados Unidos: Elsevier.

Pereira, Roberto (2006), "Violencia filio-parental: un fenómeno emergente", en Mosaico, núm. 36, España: Federación Española de Asociaciones de Terapia Familiar.

Pérez, Esther y Ruiz, Sergio (2017), "Substance Abuse as a Risk Factor for Criminal Behavior: a Systematic Review”, en Acción Psicológica, vol. 14, núm. 2, España: Universidad Nacional de Educación a Distancia.

Pierce, Mathias et al. (2017), "Insights into the link between drug use and criminality: Lifetime offending of criminally-active opiate users", en Drug and Alcohol Dependence, vol. 179, Estados Unidos: Elsevier.

Pons-Salvador, Gema (2007), "La influencia del divorcio sobre los hijos: factores moduladores del impacto psicológico", en Letras de Deusto, vol. 37, núm. 115, España: Universidad de Deusto.

Raitanen, Jenni et al. (2017), “The bullying-school shooting nexus: Bridging master narratives of mass violence with personal narratives of social exclusion”, en Deviant Behavior, vol. 40, Estados Unidos: Taylor \& Francis. 
Rechea-Alberola, Cristina y Fernández-Molina, Esther (2000), Impacto de la nueva ley penal juvenil en Castilla-La Mancha, España: Centro de Investigación en Criminología, Universidad de Castilla-La Mancha.

Rodríguez-Costas, Dámaso (1999), “Tribus urbanas violentas”, en Revista Criminalidad, núm. 51, Colombia: Policía Nacional de Colombia.

Ruiz-Callado, Raúl y Alcázar, Rafael (2019), "Las preferencias infantiles en la evaluación de la custodia compartida. Una perspectiva sociológica”, en OBETS. Revista de Ciencias Sociales, vol. 14, núm. 1, España: Universidad de Alicante.

Sánchez-Heras, Josefa et al. (2010), Manual de intervención para familias y menores con conductas de maltrato, España: Tirant lo Blanch.

Scheidell, Joy et al. (2018), "Childhood traumatic experiences and the association with marijuana and cocaine use in adolescence through adulthood”, en Addiction, vol. 113, Reino Unido: John Wiley \& Sons.

Schmidt, Fred et al. (2011), "Comparative analyses of the YLS/CMI, SAVRY, and PCL: YV in adolescent offenders: A 10-year follow-up into adulthood", en Youth Violence and Juvenile Justice, vol. 9, Estados Unidos: Sage.

Sexton, Thomas y Turner, Charles (2010), “The effectiveness of Functional Family Therapy for youth with behavioral problems in a community practice setting”, en Journal of Family Psychology, vol. 24, núm. 3, Estados Unidos: American Psychological Association.

Shepherd, Stephane et al. (2014), "The utility of the SAVRY across ethnicity in australian young offenders", en Psychological Public Policy Law, vol. 20, núm. 1, Estados Unidos: American Psychological Association.

Skara, Silvana et al. (2008), "Physical and relational aggression as predictors of drug use: Gender differences among high school students", en Addictive behaviours, vol. 33, Estados Unidos: Elsevier.

Thoumi, Francisco (2015), "The Numbers Game: Let's All Guess the Size of the Illegal Drug Industry!”, en Journal of Drug Issues, vol. 35, Estados Unidos: Sage.

Tarín, Manuel y Navarro, José Javier (2006), Adolescentes en riesgo. Casos prácticos y estrategias de intervención socioeducativa, España: CCS.

Uceda, Francesc Xavier et al. (2016), "Adolescentes y droga: su relación con la delincuencia”, en Revista de Estudios Sociales, núm. 58, Colombia: Universidad de Los Andes.

Underwood, Marion et al. (2011), "Joint trajectories for social and physical aggression as predictors of adolescent maladjustment: Internalizing symptoms, rule-breaking behaviors, and borderline and narcissistic personality features", en Development and Psychopathology, vol. 23, Estados Unidos: Cambridge University Press.

Valenzuela, Eduardo y Larroulet, Pilar (2010), "La relación droga y delito: una estimación de la fracción atribuible”, en Estudios Públicos, núm. 119, Chile: Centro de Estudios Públicos.

Vallés, Lola y Hilterman, Ed (2006), SAVRY: Manual per a la valoració estructurada de risc de violència en joves, España: Servei de Publicacions de la Generalitat de Catalunya.

Viera, Marcelo Alejandro (2017). Evaluación del riesgo de reincidencia en adolescentes en conflicto con la ley sometidos a medidas de internamiento judicial por la comisión de delitos violentos. Tesis Doctoral. Valencia (España), Universidad Católica de Valencia. 
Vilalta, Carlos (2010), "Un examen de la relación entre el consumo de sustancias y la comisión de delitos en México”, en Estudios Sociológicos, vol. 28, núm. 82, México: El Colegio de México, Centro de Estudios Sociológicos.

Walsh, Jefrey y Krienert, Jessie (2007), "Child-Parent Violence: An Empirical Analysis of Offender, Victim, and Event Characteristics in a National Sample of Reported Incidents", en Journal of Family Violence, vol. 22, núm. 7, Estados Unidos: Springer.

Walters, Glenn (2018), "Does Drug Use Inhibit Crime Deceleration or Does Crime Inhibit Drug Use Deceleration? A Test of the Reciprocal Risk Postulate of the Worst of Both Worlds Hypothesis", en Substance Use \& Misuse, vol. 53, Reino Unido: Taylor \& Francis.

Yoder, Jaime y Caserta, Deborah (2018), "Associations Between Substance Use in Commission of Sexual Crimes and Offense Characteristics Among Youth: Mitigating Effects of Substance Use Treatment", en International Journal of Offender Therapy and Comparative Criminology, vol. 62, Estados Unidos: Sage.

Zawadzki, Néstor (2007), "Violence in Childhood and Adolescence", en Pedriatrics, vol. 34, núm. 1, Estados Unidos: American Academy of Pediatrics. 


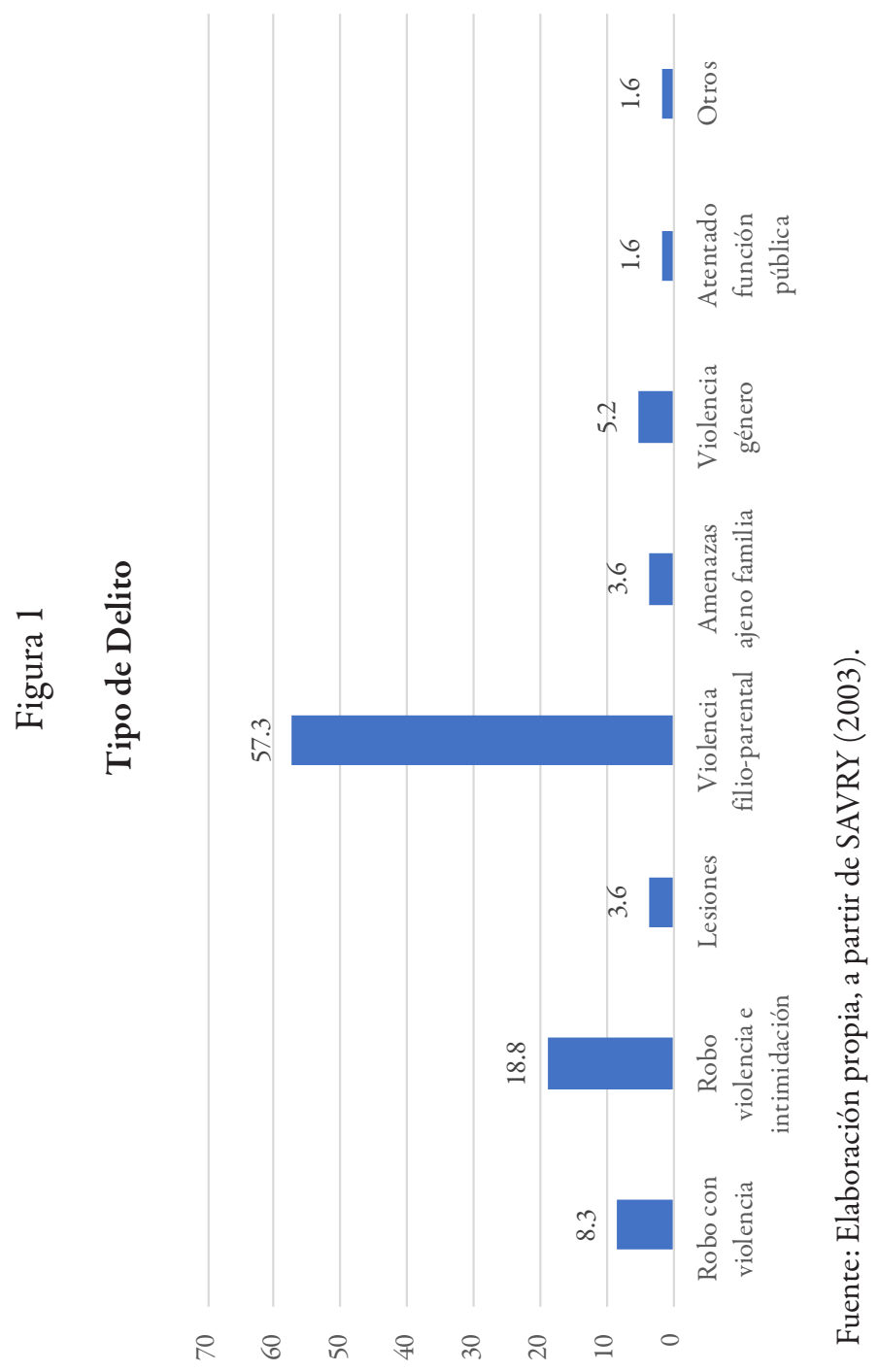

通 
Joana Calero Plaza, José Manuel Tomás, José-Javier Navarro-Pérez y Marcelo Viera. Delincuencia violenta, abuso y no consumo de drogas en adolescentes con riesgo de reincidencia

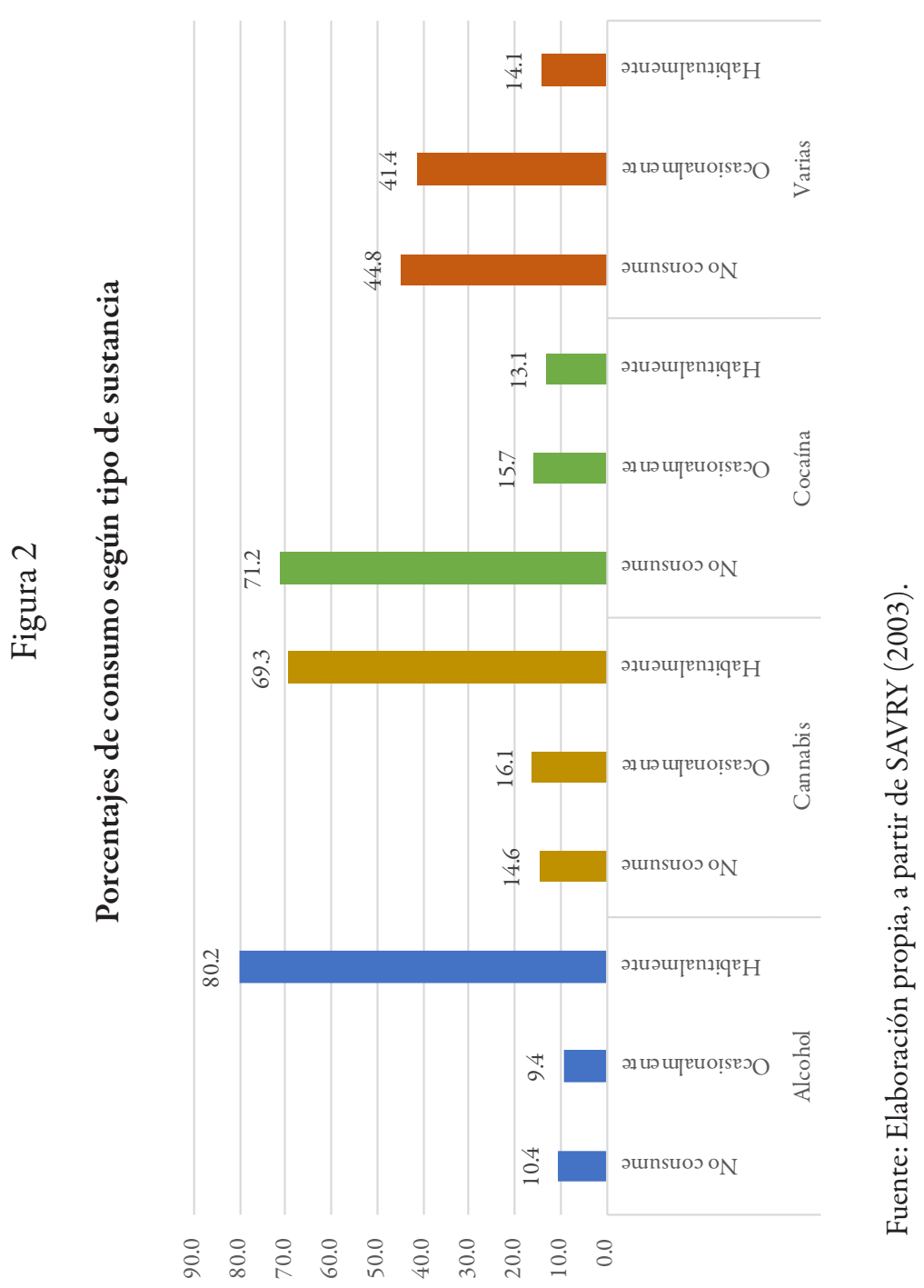




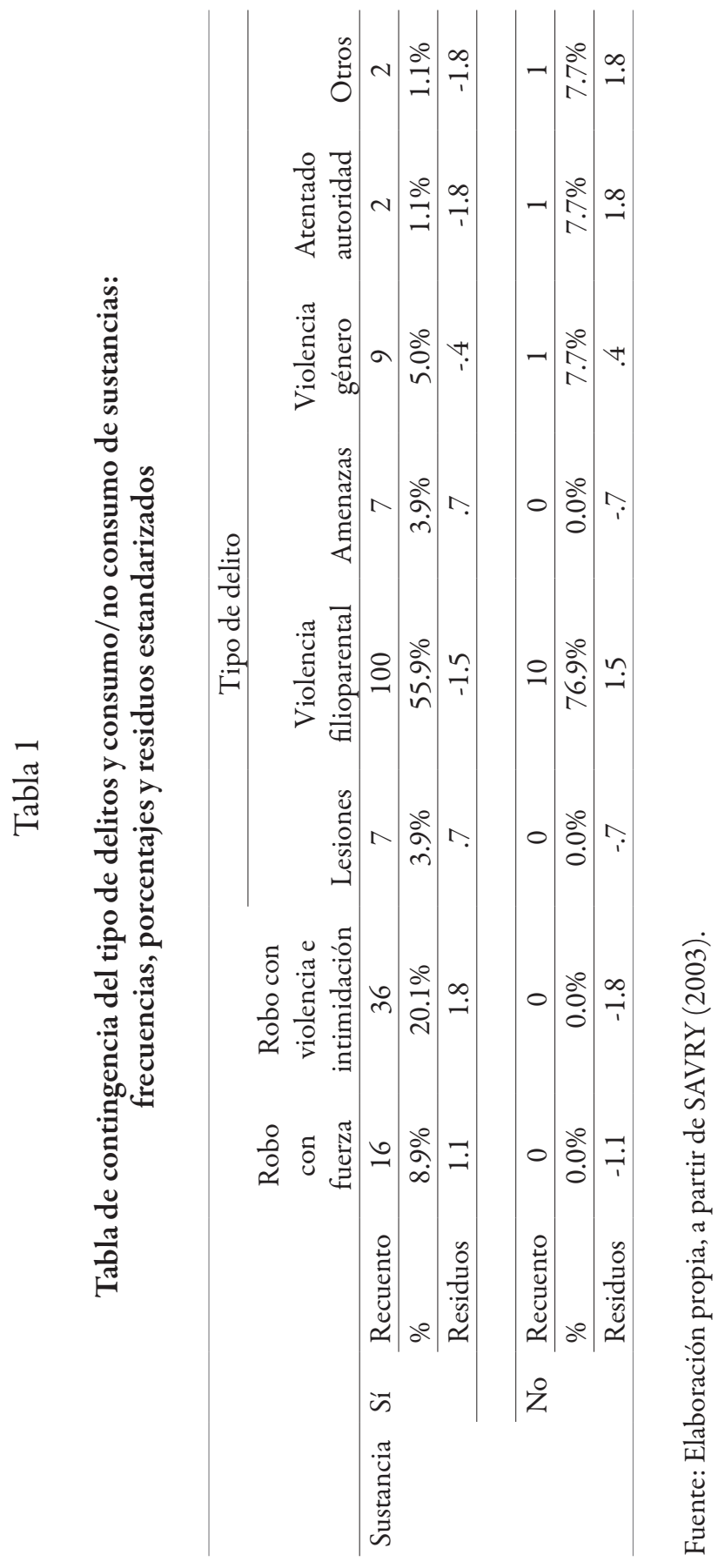


Joana Calero Plaza, José Manuel Tomás, José-Javier Navarro-Pérez y Marcelo Viera. Delincuencia violenta, abuso y no consumo de drogas en adolescentes con riesgo de reincidencia

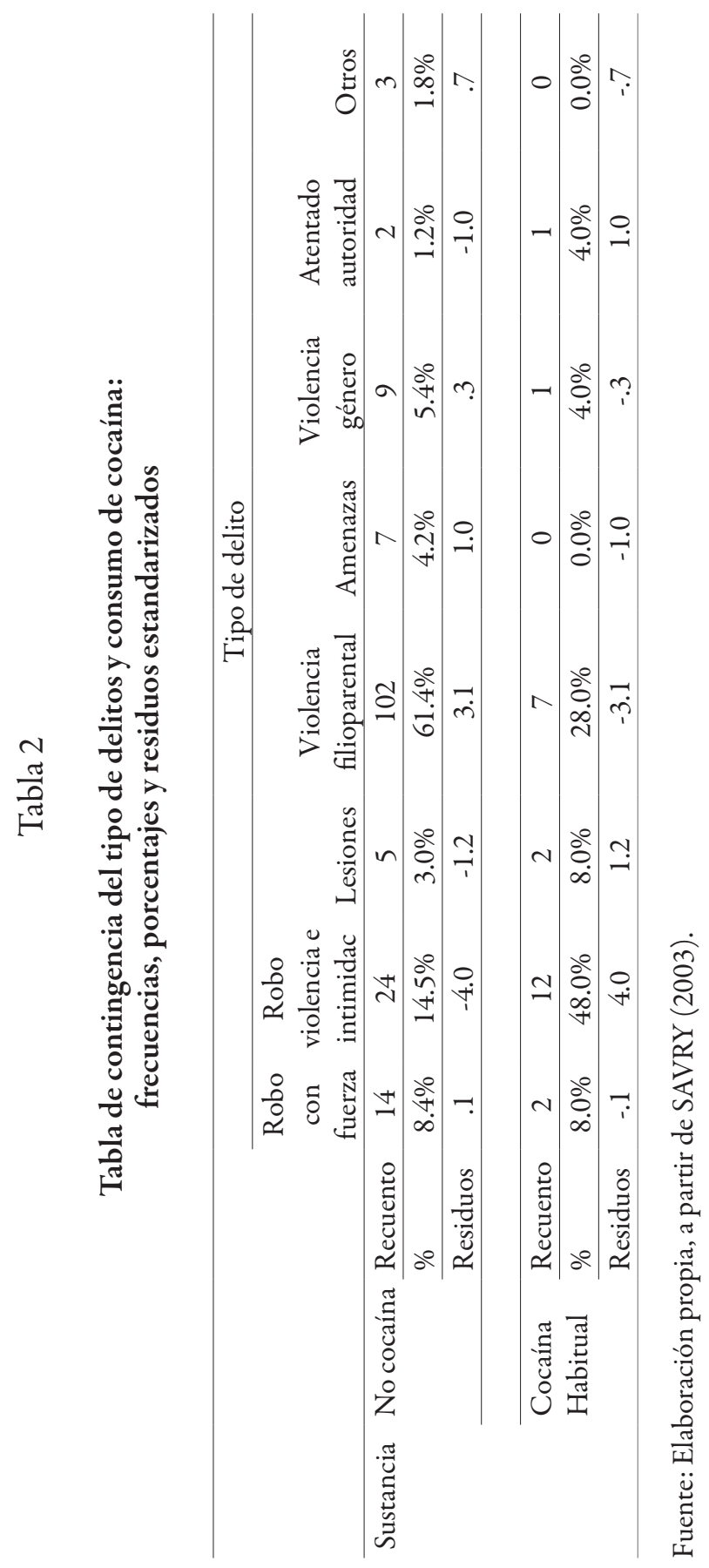




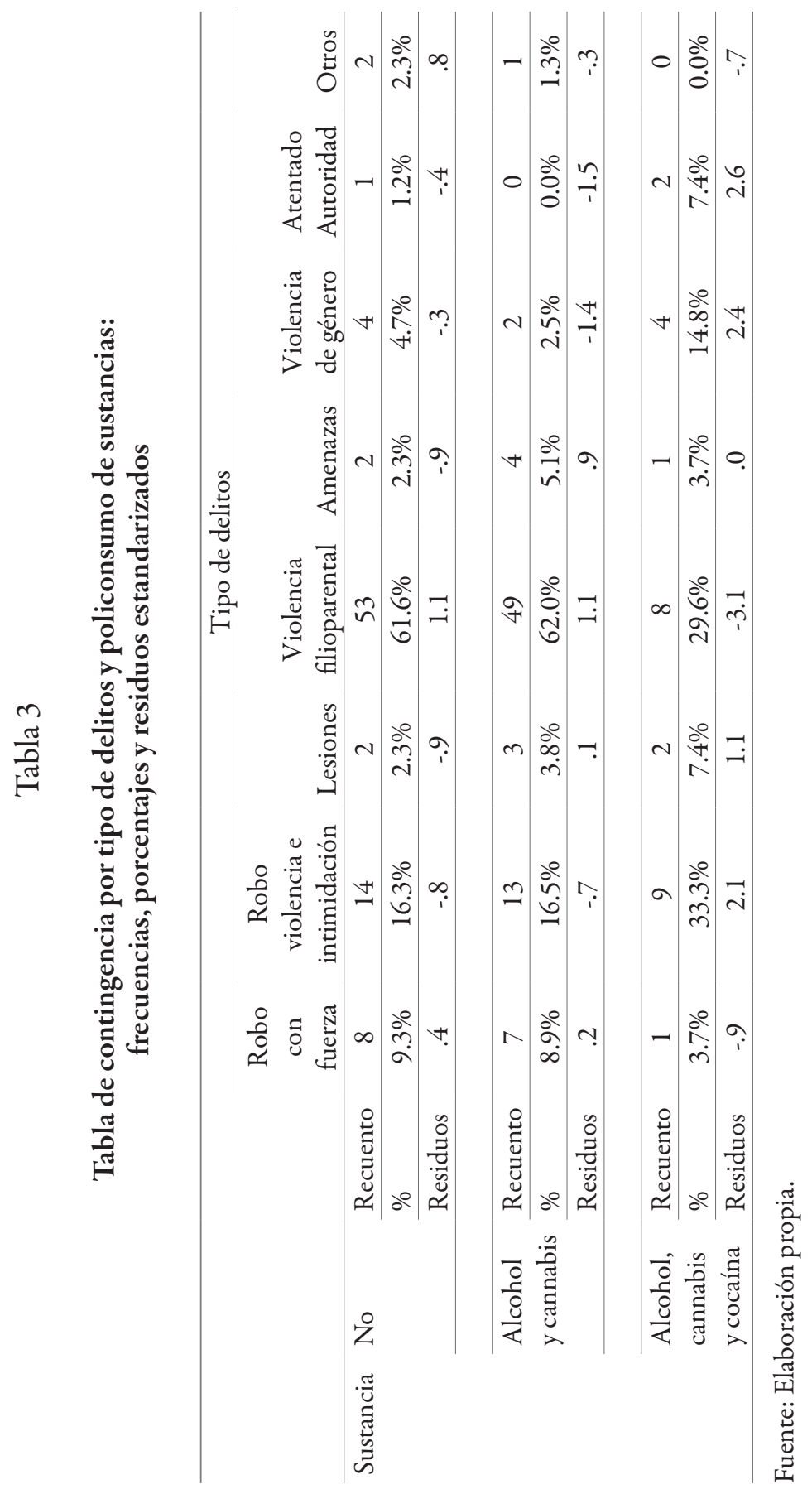


Joana Calero Plaza. Profesora del Departamento de Inclusión Educativa, Desarrollo Comunitario y Ciencias del Trabajo de la Facultad de Ciencias de la Educación, en la Universidad Católica de Valencia, España. Líneas de investigación: toxicomanías, estudios de familia y diversidad funcional. Publicaciones recientes: Calero-Plaza, Joana, Grau-Sevilla, Dolores, Martínez-Rico, Gabriel y Morales-Murillo, Catalina (2017), "Parenting Stress and Coping Strategies in Mothers of Children Receiving Early Intervention Services", en Journal of Child and Family Studies, disponible en: https:// link.springer.com/article/10.1007/s10826-017-0802-9; Calero, Joana y Cabrera, Zaray (2016), "Personas sin hogar y modelo de desarrollo positivo. Una propuesta de intervención”, en Infonova. Revista Profesional y Académica sobre las Adicciones, núm. 29, España: Asociación Dianova.

José Manuel Tomás. Catedrático de Metodología de las Ciencias del Comportamiento de la Facultad de Psicología, de la Universidad de Valencia, España. Líneas de investigación: estudios sobre metodologías de las ciencias del comportamiento, indicadores de evaluación y programas de bienestar psicosocial. Publicaciones recientes: Tomás, José Manuel, Gutiérrez, Melchor, Pastor, Ana M. y Sancho, Patricia (2020), "Perceived Social Support, School Adaptation and Adolescents' Subjective Well-Being”, en Child Indicators Research, disponible en: https://doi.org/10.1007/s12187020-09717-9; Sancho, Patricia, Tomás, José Manuel, Oliver, Amparo, Galiana, Laura y Gutiérrez, Melchor (2020), "Predicting Life satisfaction in Spanish Adults of Advancing Age Attending University Educational Programs", en Journal of Happiness Studies, núm. 21, disponible en: https:// doi.org/10.1007/s10902-019-00073-2.

José Javier Navarro-Pérez.Profesor del Departamento de Trabajo Social y Servicios Sociales de la Facultad de Ciencias Sociales e investigador del Instituto de Desarrollo Local, de la Universidad de Valencia, España. Líneas de investigación: Tecnologías de Información y Comunicación (TIC) en la adolescencia, delincuencia juvenil y salud mental en la comunidad. Publicaciones recientes: Carbonell, Ángela y Navarro-Pérez, José Javier (2019), "The care crisis in Spain: an analysis of the family care situation in mental health from a professional psychosocial perspective", en Social Work in Mental Health, disponible en: https://doi.org/10.1080/15332985.2019.1668904; Navarro-Perez, José Javier, Oliver, Amparo, Carbonell, Ángela y Schneider, Barry H. (2020), "Effectiveness of a Mobile App Intervention to Prevent Dating Violence in Residential Child Care", en Psychosocial Intervention, disponible en: https://doi.org/10.5093/pi2020a3. 
Marcelo Viera. Profesor del Departamento de Inclusión Educativa, Desarrollo Comunitario y Ciencias del Trabajo de la Facultad de Ciencias de la Educación, en la Universidad Católica de Valencia, España. Líneas de investigación: violencia juvenil, prevención y protección de menores, evaluación de programas educativos. Publicaciones recientes: Navarro-Pérez, José Javier, Viera, Marcelo, Calero, Joana y Tomás, José Manuel (2020), "Factors in Assessing Recidivism Risk in Young Offenders, en Sustainability, disponible en: https://doi.org/10.3390/su12031111; Viera, Marcelo y Nogueira, Mireia (2017), "Los/as adolescentes como participantes en el proceso de desarrollo integral de las personas con discapacidad intelectual a través del aprendizaje por servicio", en Revista de Educación Social, núm. 24, España: Consejo General de Educadoras y Educadores Sociales. 